\title{
Codoping of boron and phosphorus in silicon nanowires synthesized by laser ablation
}

\author{
N. Fukata, ${ }^{1, a)}$ M. Mitome, ${ }^{1}$ Y. Bando, ${ }^{1}$ M. Seoka, ${ }^{2}$ S. Matsushita, ${ }^{2}$ K. Murakami, ${ }^{2}$ \\ J. Chen, ${ }^{3}$ and T. Sekiguchi ${ }^{3}$ \\ ${ }_{1}^{1}$ International Center for Materials Nanoarchitectonics (MANA), National Institute for Materials Science, \\ 1-1 Namiki, Tsukuba 305-0044, Japan \\ ${ }^{2}$ Institute of Applied Physics, University of Tsukuba, 1-1-1 Tennodai, Tsukuba 305-8573, Japan \\ ${ }^{3}$ Advanced Electronic Materials Center, National Institute for Materials Science, 1-1 Namiki, \\ Tsukuba 305-0044, Japan
}

(Received 9 July 2008; accepted 3 November 2008; published online 19 November 2008)

\begin{abstract}
Codoping of boron (B) and phosphorus (P) atoms was performed during the synthesis of silicon nanowires (SiNWs) by laser ablation. The observation of a local vibrational mode of B clearly showed B doping in codoped SiNWs, while Fano broadening due to heavy B doping disappeared, indicating compensation by $\mathrm{P}$ donors. The electrospin resonance signal of conduction electrons also disappeared due to compensation by $\mathrm{B}$ acceptors. These results indicate that codoping of $\mathrm{B}$ and $\mathrm{P}$ atoms was achieved in SiNWs during laser ablation. (C) 2008 American Institute of Physics.
\end{abstract}

[DOI: $10.1063 / 1.3033226$ ]

Nanoscale silicon devices using silicon nanowires (SiNWs) have become a focus of considerable research interest since they are expected to be used in next-generation metal-oxide semiconductor field-effect transistors. Major efforts have been devoted in developing such nanoscale silicon devices. SiNWs are especially desirable due to their compatibility with current Si complementary metal-oxide semiconductor integrated circuit technology. To realize nanoscale devices such as these, the chief task is to investigate impurity doping into SiNWs since doping is a key technique for controlling the conductivity of SiNWs. A number of studies have been conducted on the synthesis and characterization of $p$-type and $n$-type SiNWs. ${ }^{1-8}$ In these studies, the effects of impurity doping were investigated mainly by electrical transport measurements. ${ }^{1-8}$ The results showed a reduction in resistivity in doped SiNWs, suggesting that SiNWs are amenable to doping with impurity atoms. We recently investigated and characterized impurity doping in SiNWs synthesized by laser ablation. 9,10 The study showed the states of dopant atoms in SiNWs such as bonding structures, sites, and electrical activities using micro-Raman scattering and electron spin resonance (ESR). ${ }^{9,10}$ The results of our microRaman scattering measurements clearly showed the presence of boron (B) local vibrational peaks and Fano broadening of Si optical phonon peaks due to heavy B doping in SiNWs. ${ }^{9}$ ESR measurements at $4.2 \mathrm{~K}$ showed the ESR signal of conduction electrons in phosphorus (P)-doped SiNWs. ${ }^{10}$ These results clearly proved that $\mathrm{B}$ and $\mathrm{P}$ atoms were doped in the crystalline Si core of SiNWs and were electrically activated in the sites. Furthermore, ESR is a sensitive method of identifying defects with dangling bonds. It is important to investigate such defects in SiNWs since they compensate carriers, which are deliberately introduced into SiNWs. Hence, Raman and ESR techniques are very useful for investigating the states of dopant atoms and defects in SiNWs.

\footnotetext{
${ }^{a)}$ Electronic mail: FUKATA.Naoki@nims.go.jp. Also at: PRESTO, Japan Science and Technology Agency, 4-1-8 Honcho Kawaguchi, Saitama 3320012, Japan.
}

In this letter, we report the effect of codoping of $\mathrm{B}$ and $\mathrm{P}$ into SiNWs. Codoping was performed during laser ablation of a Si target with $\mathrm{B}, \mathrm{P}$, and nickel (Ni) atoms. The compensation effects of each dopant atom were investigated by micro-Raman scattering and ESR measurements. The results clearly showed compensation by carriers in SiNWs.

B- or P-doped SiNWs were synthesized at $1200{ }^{\circ} \mathrm{C}$ in flowing argon (Ar) gas at 50 SCCM (SCCM denotes cubic centimeter per minute at STP) by catalytic laser ablation of a $\mathrm{Si}$ target, with $\mathrm{Ni}$ as the metal catalyst and $\mathrm{B}$ and $\mathrm{P}$ as dopants. We used nine kinds of targets, namely, $\mathrm{Si}_{89} \mathrm{Ni}_{1} \mathrm{~B}_{10}$, $\mathrm{Si}_{98} \mathrm{Ni}_{1} \mathrm{~B}_{1}, \quad \mathrm{Si}_{98.5} \mathrm{Ni}_{1} \mathrm{~B}_{0.5}, \quad \mathrm{Si}_{98.8} \mathrm{Ni}_{1} \mathrm{~B}_{0.2}, \quad \mathrm{Si}_{98.9} \mathrm{Ni}_{1} \mathrm{~B}_{0.1}$, $\mathrm{Si}_{90}\left(\mathrm{Ni}_{2} \mathrm{P}\right)_{10}, \quad \mathrm{Si}_{95}\left(\mathrm{Ni}_{2} \mathrm{P}\right)_{5}, \quad \mathrm{Si}_{99}\left(\mathrm{Ni}_{2} \mathrm{P}\right)_{1}$, and $\mathrm{Si}_{99.9}\left(\mathrm{Ni}_{2} \mathrm{P}\right)_{0.1}$. For synthesizing SiNWs codoped with $\mathrm{B}$ and $\mathrm{P}$, two targets were placed side-by-side and ablated at the center of the two targets using a frequency-doubled $\mathrm{Nd}$ :yttrium aluminum garnet laser $(532 \mathrm{~nm}, 7 \mathrm{~ns}$ pulse width, $10 \mathrm{~Hz}$, and $150 \mathrm{~mJ} /$ pulse). The details of these laser ablation procedures are reported in Ref. 11. SiNWs were directly deposited on a $\mathrm{SiO}_{2}$ substrate for micro-Raman scattering measurements. They were also directly deposited on a Si substrate, then transferred to a small quartz capsule for ESR measurements. For comparison, a $\mathrm{Si}_{99} \mathrm{Ni}_{1}$ target was also used to synthesize undoped SiNWs. Growth was conducted by the so-called vapor-liquid-solid mechanism. ${ }^{12}$ Micro-Raman scattering measurements were performed at room temperature (RT) with a $100 \times$ objective and a 532-nm excitation light. The excitation power was set at about $0.02 \mathrm{~mW}$ to avoid local heating effects from the excitation laser. ${ }^{13,14}$ ESR measurements were carried out at $4.2 \mathrm{~K}$ using an X-band ESR spectrometer with a magnetic field modulation of $100 \mathrm{kHz}$ to investigate the state of $\mathrm{P}$ donors and defects in the SiNWs. Scanning transmission electron microscopy (STEM) (Hitachi, S-5500, $30 \mathrm{kV}$ ) and transmission electron microscopy (TEM) (JEOL, JEM3100FEF: $300 \mathrm{kV}$ ) were used to observe the SiNWs and to investigate the details of their structures.

Typical STEM and TEM images of SiNWs synthesized using the $\mathrm{Si}_{95}\left(\mathrm{Ni}_{2} \mathrm{P}\right)_{5}$ and $\mathrm{Si}_{89} \mathrm{Ni}_{1} \mathrm{~B}_{10}$ targets are shown in Fig. 1(a) and a high-resolution TEM image is shown in Fig. 


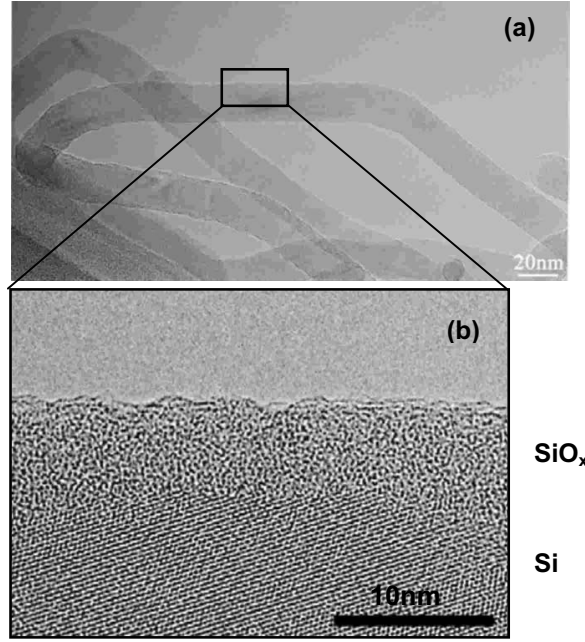

FIG. 1. Representative (a) TEM and (b) high resolution TEM images of the codoped SiNWs synthesized by laser ablation of $\mathrm{Si}_{95}\left(\mathrm{Ni}_{2} \mathrm{P}\right)_{5}$ and $\mathrm{Si}_{89} \mathrm{Ni}_{1} \mathrm{~B}_{10}$ targets.

1(b). The Si lattice fringes are seen inside the SiNW, showing that the $\mathrm{SiNW}$ is sheathed with an amorphous $\mathrm{SiO}_{x}(x$ $\leq 2)$ layer and the core comprises crystalline $\mathrm{Si}$. The average $\mathrm{Si}$ core diameter of SiNWs was about 10-15 nm.

Figure 2(a) shows Raman spectra observed for B-doped SiNWs, codoped SiNWs, undoped SiNWs, and bulk Si. The intense peak at about $520 \mathrm{~cm}^{-1}$ is due to a Si optical phonon peak. A peak at about $618 \mathrm{~cm}^{-1}$ is observed for B-doped SiNWs and codoped SiNWs. This peak has been assigned to the B local vibrational peak, ${ }^{9}$ indicating B doping into the substitutional site of the crystalline $\mathrm{Si}$ core of SiNWs. In
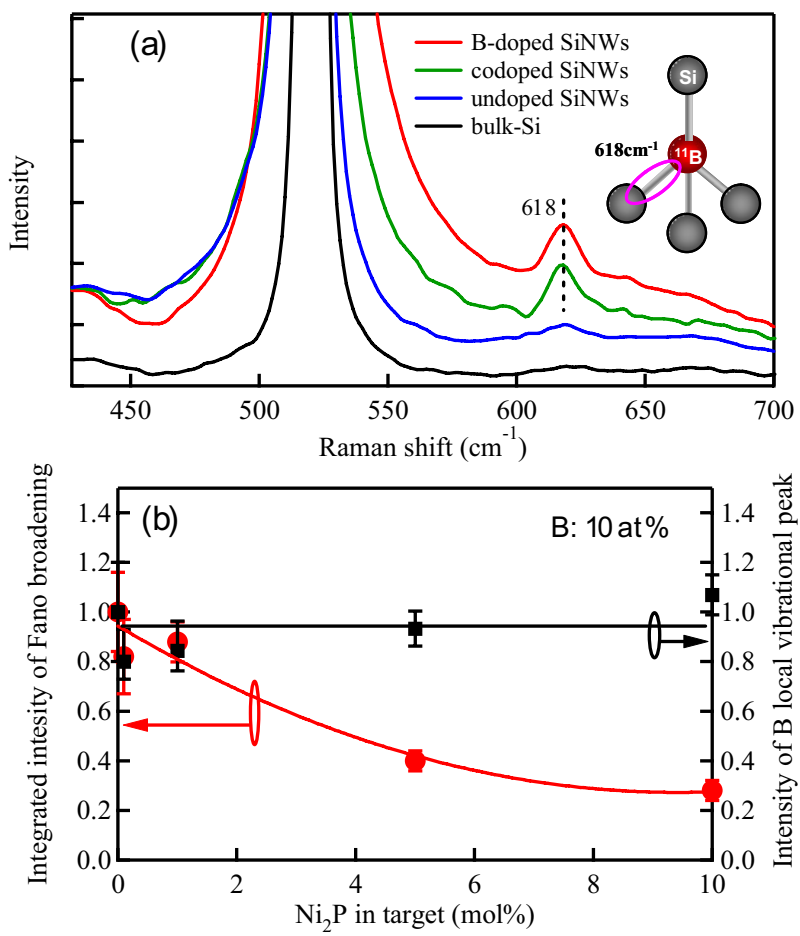

FIG. 2. (Color online) (a) Si optical phonon peaks and the B local vibrational peaks observed for B-doped SiNWs (B: 10 at\%), codoped SiNWs (B; 10 at\%, $\mathrm{Ni}_{2} \mathrm{P}: 5 \mathrm{~mol} \%$ ), undoped SiNWs, and bulk Si. (b) Dependence of Fano broadening and the $\mathrm{B}$ local vibrational peak on the content of $\mathrm{Ni}_{2} \mathrm{P}$ in the ablation targets. The micro-Raman scattering measurements were performed at an excitation power of $0.02 \mathrm{~mW}$ at RT.

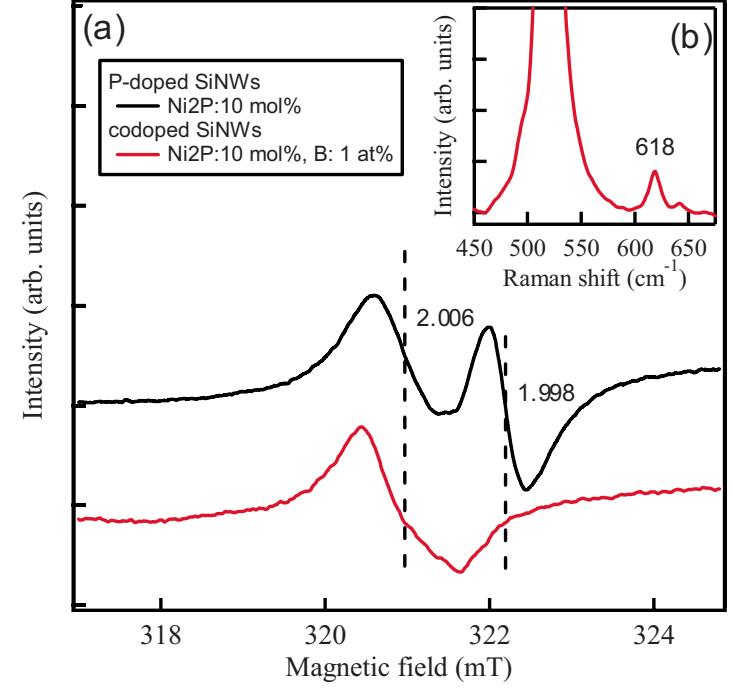

FIG. 3. (Color online) (a) ESR spectra observed for P-doped SiNWs and codoped SiNWs synthesized using $\mathrm{Si}_{90}\left(\mathrm{Ni}_{2} \mathrm{P}\right)_{10}$ and $\mathrm{Si}_{98} \mathrm{Ni}_{1} \mathrm{~B}_{1}$ targets. (b) Raman spectrum observed for the codoped SiNWs.

addition to the $\mathrm{B}$ local vibrational peak, the optical phonon peak for B-doped SiNWs and codoped SiNWs showed an asymmetric broadening to a higher wavenumber compared with that for undoped SiNWs and bulk Si. This broadening is due to the Fano effect, ${ }^{9}$ showing that B atoms are electrically activated in the crystalline $\mathrm{Si}$ core of SiNWs. The dependences on the content of $\mathrm{Ni}_{2} \mathrm{P}$ in the target are shown in Fig. 2(b). The results for codoped SiNWs show that the Fano broadening decreases on increasing the $\mathrm{Ni}_{2} \mathrm{P}$ content in the ablation targets, while the intensity of the $\mathrm{B}$ local vibrational peak remains almost constant. These clearly show that the decrease in Fano broadening is not due to the decrease in the $\mathrm{B}$ concentration but due to compensation by $\mathrm{P}$ donors.

The compensation effect in codoped SiNWs was also investigated using ESR measurements at $4.2 \mathrm{~K}$. The results are shown in Fig. 3. ESR signals with $g$-values of 1.998 and 2.006 were observed in P-doped SiNWs. The former is assigned to the ESR signal of conduction electrons in $\mathrm{Si}^{10}$ showing that $\mathrm{P}$ atoms were electrically activated in the crystalline Si core of SiNWs. The latter is assigned to the ESR signal of interfacial defects between the crystalline Si core and the surface oxide layer, which are so-called $\mathrm{P}_{b}$ type defects. ${ }^{10}$ The codoping of $\mathrm{B}$ was confirmed by observation of the B local vibrational peak at about $618 \mathrm{~cm}^{-1}$, shown in Fig. 3(b). The ESR signal of conduction electrons was significantly decreased by codoping with $\mathrm{B}$. This result clearly shows compensation by B acceptors in SiNWs.

Figure 4 shows a summary of the compensation effect in codoped $\mathrm{SiNWs}$ at constant $\mathrm{Ni}_{2} \mathrm{P}$ content $(10 \mathrm{~mol} \%)$ in the ablation targets. The result clearly shows the codoping of $\mathrm{B}$ and P in SiNWs, resulting in compensation by B acceptors. The ESR signal of conduction electrons drastically decreased on increasing the $\mathrm{B}$ concentration and was not observed above 1 at. $\%$ of $\mathrm{B}$, while the $\mathrm{B}$ local vibrational peak gradually increased. This can be explained by the difference in the concentrations of $\mathrm{P}$ and $\mathrm{B}$ in the codoped SiNWs, i.e., the concentration of electrically active $\mathrm{P}$ donors is lower than that of B acceptors.

A theoretical calculation shows that the formation energy for P doping in SiNWs is smaller than that for B doping, meaning that the solubility of $\mathrm{P}$ is greater than that of $\mathrm{B} .{ }^{15}$ 


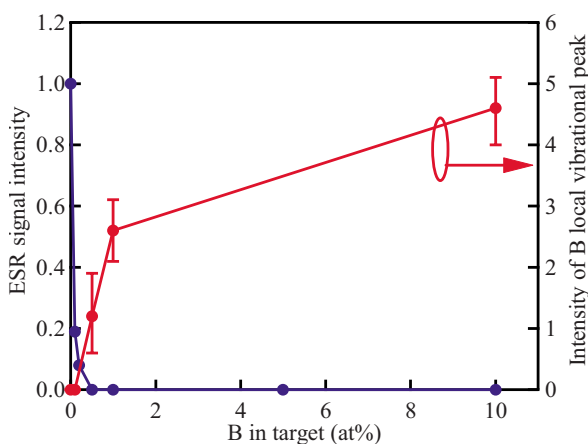

FIG. 4. (Color online) (a) Dependence of the ESR signal intensity of the conduction electrons and the intensity of the $\mathrm{B}$ local vibrational peak on the content of B in targets.

This is in agreement with the experimental results reported for bulk Si. For example, according to a previous study, the solubility of $\mathrm{P}$ atoms in bulk $\mathrm{Si}$ is about $10^{21} \mathrm{~cm}^{-3}$ at $1200{ }^{\circ} \mathrm{C}$, while for $\mathrm{B}$ it is about $5 \times 10^{20} \mathrm{~cm}^{-3} \cdot{ }^{16}$ On the other hand, the result in Fig. 4 suggests that the concentration of electrically active $\mathrm{P}$ donors is much lower than that of B acceptors, so to explain the result in Fig. 4, another reason needs to be considered. The tendency shown in Fig. 4 is in agreement with experimental conductivity measurements for SiNWs, showing that the concentration of free carriers is much lower for P doping than B doping for the same impurity density. ${ }^{1,17}$ This suggests that the electrical activation ratio of the dopant atoms has to be considered in SiNWs. The electrical activity is related to the states of $\mathrm{P}$ and $\mathrm{B}$ atoms in SiNWs. According to recent theoretical calculations, the segregation energy of $\mathrm{P}$ atoms at the surface of a SiNW is significantly larger than that of $\mathrm{B}$ atoms, with the result that $\mathrm{P}$ segregation is more pronounced than B segregation. ${ }^{16,18}$ The calculation also shows that the larger segregation energy for $\mathrm{P}$ atoms causes the attraction of $\mathrm{P}$ atoms to dangling bonds, and they become electrically inactive at the trapping sites. These dangling bonds are present at the interface between the surface oxide layer and the crystalline Si core of SiNWs. In fact, the ESR signal of interfacial defects can be observed, as shown in Fig. 3. Based on these results, the difference in segregation behaviors is considered to be one of the reasons for the concentration of electrically active $\mathrm{P}$ donors being markedly lower than that of B acceptors.
In conclusion, codoping of $\mathrm{B}$ and $\mathrm{P}$ dopant atoms in SiNWs during laser ablation was confirmed by ESR and Raman scattering measurements. The compensation effect was clearly observed by the change in the ESR signal of conduction electrons and Fano broadening of the Si optical phonon peak. These results suggest that the concentration of $\mathrm{P}$ donors tends to be lower than that of $\mathrm{B}$ acceptors. This can be explained by the difference in segregation behaviors between $\mathrm{P}$ and $\mathrm{B}$ in SiNWs.

This study was supported by the Japan Science and Technology Agency (JST). This work was also partly supported by the Innovation Research Project on Nanoelectronics Materials and Structures, the Murata Science Foundation, and the World Premier International Research Center Initiative (WPI Initiative) on Materials Nanoarchitectonics, MEXT, Japan.

${ }^{1}$ Y. Cui, X. Duan, J. Hu, and C. M. Lieber, J. Phys. Chem. B 104, 5213 (2000)

${ }^{2}$ Y. Cui and C. M. Lieber, Science 291, 851 (2001).

${ }^{3}$ D. D. D. Ma, C. S. Lee, and S. T. Lee, Appl. Phys. Lett. 79, 2468 (2001).

${ }^{4}$ K. K. Lew, L. Pan, T. E. Bogart, S. M. Dilts, E. C. Dickey, J. M. Redwing, Y. Wang, M. Cabassi, T. S. Mayer, and S. W. Novak, Appl. Phys. Lett. 85, 3101 (2004)

${ }^{5}$ G. Zheng, W. Lu, S. Jin, and C. M. Lieber, Adv. Mater. (Weinheim, Ger.) 16, 1890 (2004).

${ }^{6}$ L. Pan, K. K. Lew, J. M. Redwing, and E. C. Dickey, J. Cryst. Growth 277, 428 (2005).

${ }^{7}$ Y. Wang, K. K. Lew, T. T. Ho, L. Pan, S. W. Novak, E. C. Dickey, J. M. Redwing, and T. S. Mayer, Nano Lett. 5, 2139 (2005).

${ }^{8}$ C. Yang, Z. Zhong, and C. M. Lieber, Science 310, 1304 (2005).

${ }^{9}$ N. Fukata, J. Chen, T. Sekiguchi, N. Okada, K. Murakami, T. Tsurui, and S. Ito, Appl. Phys. Lett. 89, 203109 (2006).

${ }^{10}$ N. Fukata, J. Chen, T. Sekiguchi, S. Matsushita, T. Oshima, N. Uchida, K. Murakami, T. Tsurui, and S. Ito, Appl. Phys. Lett. 90, 153117 (2007).

${ }^{11}$ N. Fukata, T. Oshima, K. Murakami, T. Kizuka, T. Tsurui, and S. Ito, Appl. Phys. Lett. 86, 213112 (2005).

${ }^{12}$ R. S. Wagner and W. C. Ellis, Appl. Phys. Lett. 4, 89 (1964).

${ }^{13}$ S. Piscanec, M. Cantoro, A. C. Ferrari, J. A. Zapien, Y. Lifshitz, S. T. Lee, S. Hofmann, and J. Robertson, Phys. Rev. B 68, 241312(R) (2003).

${ }^{14}$ N. Fukata, T. Oshima, N. Okada, T. Kizuka, T. Tsurui, S. Ito, and K. Murakami, J. Appl. Phys. 100, 024311 (2006).

${ }^{15}$ H. Peelaers, B. Partoens, and F. M. Peeters, Nano Lett. 6, 2781 (2006).

${ }^{16}$ F. A. Trumbore, Bell Syst. Tech. J. 39, 205 (1960).

${ }^{17}$ J.-Y. Yu, S.-W. Chung, and J. R. Heath, J. Phys. Chem. B 104, 11864 (2000).

${ }^{18}$ M. V. Fernández-Serra, Ch. Adessi, and X. Blase, Phys. Rev. Lett. 96 166805 (2006). 